\title{
On almost even arithmetical functions via orthonormal systems on Vilenkin groups
}

by

\author{
G. GÁt (Nyiregyháza)
}

1. Introduction, preliminaries. Let $k \in \mathbb{N}$. An arithmetical function $f: \mathbb{N} \rightarrow \mathbb{C}$ is called even $\bmod k$ if $f((n, k))=f(n)$ for all $n \in \mathbb{N}$. Define $D:=\{f \mid f: \mathbb{N} \rightarrow \mathbb{C}\}, D^{b}:=\{f \in D, f$ bounded $\}, \mathcal{B}_{k}:=\{f \in D \mid$ $f$ even $\bmod k\}, \mathcal{B}:=\bigcup_{k \in \mathbb{N}} \mathcal{B}_{k}$. Then $\mathcal{B}$ is the $\mathbb{C}$-algebra (with pointwise addition and multiplication) of even functions, and $D^{b}$ with the "uniform norm" $\|f\|_{u}:=\sup _{n \in \mathbb{N}}|f(n)|$ is a complex Banach algebra. The limit (if it exists)

$$
M(f):=\lim _{x \rightarrow \infty} x^{-1} \sum_{1 \leq j \leq x} f(j)
$$

is called the mean value of $f$.

$$
\text { If } \begin{aligned}
f: \mathbb{N} & \rightarrow \mathbb{R} \text {, then } \\
\bar{M}(f) & :=\limsup _{x \rightarrow \infty} x^{-1} \sum_{1 \leq j \leq x} f(j), \quad \underline{M}(f):=\liminf _{x \rightarrow \infty} x^{-1} \sum_{1 \leq j \leq x} f(j)
\end{aligned}
$$

are called the upper and the lower mean values of $f$, respectively.

From now on throughout this paper we suppose that $1 \leq q<\infty$. Then the upper mean value gives rise to a seminorm $\|f\|_{q}:=\left\{\bar{M}\left(|f|^{q}\right)\right\}^{1 / q}$ on the linear space $\left\{f \in D \mid\|f\|_{q}<\infty\right\}$. We denote by $\mathcal{B}^{q}$ the closure of $\mathcal{B}$ in $\left\{f \in D \mid\|f\|_{q}<\infty\right\}$ relative to the topology defined by $\|\cdot\|_{q}$. Functions in $\mathcal{B}^{q}$ are called $\mathcal{B}^{q}$ almost even arithmetical functions. Denote by $\mathcal{B}^{u}$ the closure of $\mathcal{B}$ in $D^{b}$ relative to the topology defined by $\|\cdot\|_{u}$. Every $f \in \mathcal{B}^{u}$ is called a uniform almost even arithmetical function.

Let $m:=\left(m_{0}, m_{1}, \ldots\right)$ denote a sequence of positive integers not less than 2. Denote by $Z_{m_{j}}:=\left\{0,1, \ldots, m_{j}-1\right\}$ the additive group of integers modulo $m_{j}\left(j \in \mathbb{N}_{0}\right)$. Define the group $G_{m}$ as the cartesian product of the discrete cyclic groups $Z_{m_{j}}$,

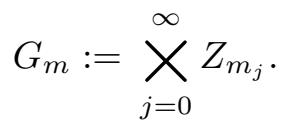


The elements of $G_{m}$ can be represented by sequences $x:=\left(x_{0}, x_{1}, \ldots\right)\left(x_{j} \in\right.$ $\left.Z_{m_{j}}\right)$. It is easy to give a base for the neighborhoods of $G_{m}$ :

$$
\begin{aligned}
& I_{0}(x):=G_{m}, \\
& I_{n}(x):=\left\{y \in G_{m} \mid y_{0}=x_{0}, \ldots, y_{n-1}=x_{n-1}\right\}
\end{aligned}
$$

for $x \in G_{m}, n \in \mathbb{N}$. Define $I_{n}:=I_{n}(0)$ for $n \in \mathbb{N}_{0}$. Then $I_{n}$ is a subgroup of $G_{m}\left(n \in \mathbb{N}_{0}\right)$. Moreover, $G_{m}$ is a compact zero-dimensional abelian group. The direct product $\mu$ of the measures

$$
\mu_{k}(\{j\}):=m_{k}^{-1} \quad\left(j \in Z_{m_{k}}, k \in \mathbb{N}_{0}\right)
$$

is the Haar measure on $G_{m}$ with $\mu\left(G_{m}\right)=1$.

Define the generalized powers by $M_{0}:=1, M_{k+1}:=m_{k} M_{k}\left(k \in \mathbb{N}_{0}\right)$. Then every nonnegative integer $n$ can be uniquely expressed as $\sum_{j=0}^{\infty} n_{j} M_{j}$, where $n_{j} \in Z_{m_{j}}\left(j \in \mathbb{N}_{0}\right)$ and only a finite number of $n_{j}$ 's differ from zero.

Define on $G_{m}$ the generalized Rademacher functions in the following way:

$$
r_{k}(x):=\exp \left(2 \pi i x_{k} / m_{k}\right) \quad\left(i:=(-1)^{1 / 2}, x \in G_{m}, k \in \mathbb{N}_{0}\right) .
$$

It is known that the functions

$$
\psi_{n}:=\prod_{k=0}^{\infty} r_{k}^{n_{k}} \quad\left(n \in \mathbb{N}_{0}\right)
$$

on $G_{m}$ are elements of the character group of $G_{m}$, and all the elements of the character group are of this form. The system $\left(\psi_{n} \mid n \in \mathbb{N}_{0}\right)$ is called a Vilenkin system and $G_{m}$ a Vilenkin group. For more details on Vilenkin analysis see e.g. $[1,14,17,18]$.

Let $\mathcal{A}_{n}\left(n \in \mathbb{N}_{0}\right)$ be the $\sigma$-algebra generated by the cosets $I_{n}(z)(z \in$ $\left.G_{m}\right)$. Let $\alpha_{j}^{k}, \alpha_{n}(k, j, n \in \mathbb{N})$ be functions satisfying the following conditions:

(i) $\alpha_{j}^{k}: G_{m} \rightarrow \mathbb{C}$ is $\mathcal{A}_{j}$-measurable $\left(k, j \in \mathbb{N}_{0}\right)$,

(ii) $\left|\alpha_{j}^{k}\right|=\alpha_{0}^{k}=\alpha_{k}^{0}=1\left(k, j \in \mathbb{N}_{0}\right)$,

(iii) $\alpha_{n}:=\prod_{j=0}^{\infty} \alpha_{j}^{j(n)}\left(n \in \mathbb{N}_{0}, j(n):=\sum_{k=j}^{\infty} n_{k} M_{k}\right)$.

Let $\phi_{n}:=\psi_{n} \alpha_{n}\left(n \in \mathbb{N}_{0}\right)$. A function system $\left\{\phi_{n} \mid n \in \mathbb{N}_{0}\right\}$ of this type is called a $\psi \alpha$ system on the Vilenkin group $G_{m}$. We can identify $G_{m}$ with the unit interval $[0,1)$ by associating with each $\left(x_{0}, x_{1}, \ldots\right)=x \in G_{m}$ the point $\sum_{j=0}^{\infty} x_{j} M_{j+1}^{-1} \in[0,1)$.

If we disregard the countable set of $m$-rationals,

$$
\mathbb{Q}_{m} \cap(0,1) \quad\left(\mathbb{Q}_{m}:=\left\{x \in G_{m} \mid \exists j \in \mathbb{N}_{0}: \forall k \geq j, k \in \mathbb{N}_{0}, x_{k}=0\right\}\right),
$$

then this mapping is one-one and measure preserving. Every $y \in \mathbb{Q}_{m} \cap(0,1)$ has a duplicate in $G_{m}$, one of them has a finite and the other an infinite representation of the form $y=\sum y_{j} M_{j+1}^{-1}$. 
Now we introduce a special kind of $\psi \alpha$ systems on the Vilenkin group $G_{m}$ (which is identified with $[0,1)$ in the way described above).

If $n \in \mathbb{N}_{0}, n=\sum_{j=0}^{\infty} n_{j} M_{j}$, then let

$$
\check{n}:=\sum_{j=0}^{\infty} n_{j} M_{j+1}^{-1} \in[0,1)
$$

(of course only a finite number of $n_{j}$ 's are not zero).

If $x \in G_{m}, A \in \mathbb{N}_{0}$, then let $\sigma_{A} x:=\sum_{j=0}^{A} x_{j} M_{j} \in \mathbb{N}_{0}$. Suppose that $n<M_{A+1}$ for some $A \in \mathbb{N}_{0}$. Then $\check{n}=\sum_{j=0}^{A} n_{j} M_{j+1}^{-1}$. Then the function $\mathbb{N}_{0} \ni k \rightarrow \exp \left(2 \pi i \check{n} \sigma_{A+k} x\right)\left(n, A \in \mathbb{N}_{0}, x \in G_{m}\right.$ fixed) is constant, because

$$
\begin{aligned}
\exp \left(2 \pi i \check{n} \sigma_{A+k} x\right) \\
=\exp \left\{2 \pi i\left(\frac{n_{0}}{M_{1}}+\frac{n_{1}}{M_{2}}+\ldots+\frac{n_{A}}{M_{A+1}}\right)\right. \\
\left.\quad \times\left(x_{0}+x_{1} M_{1}+\ldots+x_{A} M_{A}+x_{A+1} M_{A+1}+\ldots+x_{A+k} M_{A+k}\right)\right\} \\
=\exp \left(2 \pi i\left(\frac{n_{0}}{M_{1}}+\ldots+\frac{n_{A}}{M_{A+1}}\right)\left(x_{0}+x_{1} M_{1}+\ldots+x_{A} M_{A}\right)\right) \\
=\exp \left(2 \pi i \check{n} \sigma_{A} x\right) .
\end{aligned}
$$

Thus the definition

$$
\kappa_{n}(x):=\lim _{k \rightarrow \infty} \exp \left(2 \pi i \check{n} \sigma_{k} x\right) \quad\left(n \in \mathbb{N}_{0}, x \in G_{m}\right)
$$

makes sense.

$\kappa_{n}(x)$ can also be denoted as $\exp (2 \pi i \check{n} \sigma x)$. The system $\left\{\kappa_{n} \mid n \in \mathbb{N}_{0}\right\}$ on $G_{m}$ is a $\psi \alpha$ system.

Indeed, suppose that $M_{A} \leq n<M_{A+1}, x \in G_{m}, 1 \leq A$. Then

$$
\begin{aligned}
\kappa_{n}(x)= & \exp \left(2 \pi i\left(\frac{n_{0}}{m_{0}}+\ldots+\frac{n_{A}}{m_{0} \ldots m_{A}}\right) x_{0}\right) \\
& \times \exp \left(2 \pi i\left(\frac{n_{1}}{m_{1}}+\ldots+\frac{n_{A}}{m_{1} \ldots m_{A}}\right) x_{1}\right) \ldots \exp \left(2 \pi i \frac{n_{A}}{m_{A}} x_{A}\right) \\
= & r_{0}^{n_{0}}(x) \ldots r_{A}^{n_{A}}(x) \prod_{j=0}^{A-1} \exp \left(2 \pi i\left(\frac{n_{j+1}}{m_{j} m_{j+1}}+\ldots+\frac{n_{A}}{m_{j} \ldots m_{A}}\right)\right) .
\end{aligned}
$$

That is, in this case

$$
\alpha_{j}^{j(n)}=\exp \left(2 \pi i x_{j-1}\left(\frac{n_{j}}{m_{j-1} m_{j}}+\ldots+\frac{n_{A}}{m_{j-1} \ldots m_{A}}\right)\right) \quad(1 \leq j \in \mathbb{N}) .
$$

Of course if $A=0$, then the product $\prod_{j=0}^{A-1}$ is equal to 1 , and for $n<M_{0}$, i.e. $n=0, \kappa_{0}(x)=1=\psi_{0}(x) \alpha_{0}(x)$. We have proved that $\left\{\kappa_{n} \mid n \in \mathbb{N}_{0}\right\}$ is 
a $\psi \alpha$ system on $G_{m}$.

The Fourier coefficients of $f \in L^{1}\left(G_{m}\right)$ with respect to the $\psi \alpha$ system $\kappa$ are defined by

$$
\hat{f}(k)=\hat{f}^{\kappa}(k):=\int_{G_{m}} f(x) \bar{\kappa}_{k}(x) d \mu(x) \quad\left(k \in \mathbb{N}_{0}\right) .
$$

The Dirichlet kernels are given by

$$
D_{n}(x, y)=D_{n}^{\kappa}(x, y):=\sum_{j=0}^{n-1} \kappa_{j}(x) \bar{\kappa}_{j}(y) \quad\left(x, y \in G_{m}, n \in \mathbb{N}\right) .
$$

The $n$th partial sum of the Fourier series of $f \in L^{1}\left(G_{m}\right)$ (with respect to the $\psi \alpha$ system $\kappa)$ is

$$
S_{n} f(x)=S_{n}^{\kappa} f(x):=\sum_{j=0}^{n-1} \hat{f}^{\kappa}(j) \kappa_{j}(x) \quad\left(x \in G_{m}, n \in \mathbb{N}\right) .
$$

We give some examples of Vilenkin groups $G_{m}$.

If each $m_{j}\left(j \in \mathbb{N}_{0}\right)$ equals 2 , then $G_{m}$ is called the Walsh-Paley group. The character system of this special Vilenkin group is the set of Walsh functions. The Walsh functions have three most studied enumerations, namely the original Walsh, the Walsh-Kaczmarz and the Walsh-Paley one. The last one coincides with the ordering used in this paper. For more details on Walsh functions see e.g. the recent book of F. Schipp, W. R. Wade, P. Simon and J. Pál ([14]).

If the sequence $m$ is bounded, then $G_{m}$ is called a bounded Vilenkin group. Most of the results on the Walsh-Paley group also hold for bounded Vilenkin groups. But if the sequence $m$ is not bounded, then the situation changes. There are many theorems which hold on bounded Vilenkin groups but fail to hold on unbounded ones. For more details on Vilenkin groups see $[1]$.

Define the dyadic addition of $k, n \in \mathbb{N}_{0}$ as follows:

$$
k \oplus n:=\sum_{j=0}^{\infty}\left(\left(k_{j}+n_{j}\right) \bmod m_{j}\right) M_{j} .
$$

Since $\left(\kappa_{n}\right)$ is a $\psi \alpha$ system, Theorems $1,2,3$ below are direct applications of similar ones in [4].

THEOREM 1. The system $\left\{\kappa_{n}\right\}_{n \geq 0}$ is orthonormal on $G_{m}$, that is,

$$
\int_{G_{m}} \kappa_{k}(x) \bar{\kappa}_{n}(x) d \mu(x)=\delta_{k, n} \quad \text { (the Kronecker delta), }
$$

$k, n \in \mathbb{N}_{0}$, and complete in $L^{1}\left(G_{m}\right)$. 
Theorem 2. If $t \in \mathbb{N}_{0}$ and $x, y \in G_{m}$, then

$$
D_{M_{t}}(x, y)= \begin{cases}0 & \text { if } x \notin I_{t}(y), \\ M_{t} & \text { if } x \in I_{t}(y) .\end{cases}
$$

Theorem 3. If $f \in L^{q}\left(G_{m}\right)(q \geq 1)$ and $n \in \mathbb{N}_{0}$, then

$$
\left(\int_{G_{m}}\left|S_{M_{n}} f\right|^{q}\right)^{1 / q} \leq A_{q}\left(\int_{G_{m}}|f|^{q}\right)^{1 / q}=: A_{q}\|f\|_{L^{q}},
$$

where the constant $A_{q}$ does not depend on $f$.

Theorem 4 can be proved by a slight modification of F. Schipp's method [12].

TheOREM 4. If $f \in L^{q}\left(G_{m}\right)(q>1)$ and $n \in \mathbb{N}$, then

$$
\left\|S_{n} f\right\|_{L^{q}} \leq A_{q}\|f\|_{L^{q}}
$$

for some $A_{q}$ depending only on $q$.

Next we deal with the relation between almost even arithmetical functions and Vilenkin analysis. John Knopfmacher has also been concerned with Fourier analysis of arithmetical functions; it is worthwhile to compare his theory and the analytical methods on Vilenkin groups (see [9] and [10]).

2. Results on $\mathcal{B}^{q}$ and $\mathcal{B}^{u}$. From now on throughout this paper the following condition will hold for the sequence $\left\{m_{j}\right\}$ :

For all $k \in \mathbb{N}$ there exists an $n=n(k) \in \mathbb{N}$ such that $k \mid M_{n}$.

A Vilenkin group $G_{m}$ generated by a sequence $m$ of this kind is called $R$ (Ramanujan)-Vilenkin.

TheOREM 5. If $f \in \mathcal{B}^{u}$, then there exists a unique continuous $f^{*}: G_{m} \rightarrow$ $\mathbb{C}$ such that $f^{*}(\check{n})=f(n)$ for all $n \in \mathbb{N}$ and $M(f)=\int_{G_{m}} f^{*} d \mu$.

Theorem 6 (compare Knopfmacher [9]). If $f \in \mathcal{B}^{q}(q \geq 1)$, then there exists an $f^{*}: G_{m} \rightarrow \mathbb{C}$ such that

$$
\left\|f^{*}\right\|_{L^{q}}=\|f\|_{q}, \quad f_{n} \stackrel{\|\cdot\|_{q}}{\longrightarrow} f \Leftrightarrow f_{n}^{*} \stackrel{\|\cdot\|_{L^{q}}}{\longrightarrow} f^{*} .
$$

$f^{*}$ is unique (in the sense of equality $\mu$-almost everywhere).

Theorem 7. If $f, g \in \mathcal{B}^{q}(q \geq 1)$ and $|g|<c$, then $f g \in \mathcal{B}^{q}$ and $(f g)^{*}=f^{*} g^{*} \mu$-a.e.

Theorem 8. If $n \in \mathbb{N}_{0}$ and $g(j)=\exp (2 \pi i \check{n} j)(j \in \mathbb{N})$, then

$$
g^{*}(x)=\exp (2 \pi i \check{n} \sigma x)=\kappa_{n}(x) \quad\left(x \in G_{m}\right) .
$$


Theorem 9. If $f \in \mathcal{B}^{q}(q \geq 1)$ and $k \in \mathbb{N}_{0}$, then

$$
M\left(f e^{-2 \pi i \breve{k}}\right)=\int_{G_{m}} f^{*}(x) \bar{\kappa}_{k}(x) d \mu(x)=\left(\hat{f}^{*}\right)(k) .
$$

The Ramanujan sum $c_{r}$ is defined as

$$
c_{r}(n):=\sum_{\substack{a=1 \\(a, r)=1}}^{r} \exp (2 \pi i(a / r) n) \quad(r, n \in \mathbb{N}) .
$$

If $r \mid k$, then $c_{r} \in \mathcal{B}_{k}$. Cohen [2] and later Schwarz and Spilker [15] proved that $f \in \mathcal{B}_{k}$ implies

$$
f=\sum_{r \mid k} \alpha_{r} c_{r}, \quad \alpha_{r}=\varphi^{-1}(r) k^{-1} \sum_{n=1}^{k} f(n) c_{r}(n),
$$

where the coefficients $\alpha_{r}$ are uniquely determined and $\varphi$ is the Euler function. Define

$$
\begin{aligned}
& \mathcal{L}^{q}\left(G_{m}\right):=\left\{f \in L^{q}\left(G_{m}\right) \mid\right. \\
&\text { there exists a } \left.g \in \mathcal{B}^{q} \text { such that } g^{*}=f \mu \text {-a.e. }\right\} \quad(q \geq 1), \\
& \hat{g}^{\mathrm{R}}(r):=\varphi^{-1 / 2}(r) \int_{G_{m}} g^{*} \bar{c}_{r}^{*}, \quad K_{k} g:=\sum_{r \mid k} \varphi^{-1}(r) M\left(g \bar{c}_{r}\right) c_{r}
\end{aligned}
$$

$\left(r, k \in \mathbb{N}, g \in \mathcal{B}^{q}, q \geq 1\right)$.

It is not difficult to prove that on each $\mathrm{R}$-Vilenkin group $G_{m}$ the set of $m$-rationals $\mathbb{Q}_{m}$ equals the set of "ordinary" rationals $\mathbb{Q}$. This yields

Proposition 10.

$$
c_{r}^{*}=\sum_{\substack{a=1 \\(a, r)=1}}^{r} \kappa_{(a / r)^{\vee}} \quad\left(1<r \in \mathbb{N}, c_{1}^{*}=\kappa_{0}\right)
$$

on $R$-Vilenkin groups.

Proposition 11. Let $f^{*} \in \mathcal{L}^{q}\left(G_{m}\right)(q \geq 1)$. Then each member of the set $\left\{\hat{f}^{*}(n) \mid \check{n}=a / r,(a, r)=1, a \in\{1, \ldots, r\}\right\}$ equals $\hat{f}^{\mathrm{R}}(r) \varphi^{-1 / 2}(r)$.

Corollaries 12 and 13 below are obvious consequences of Theorems 1, 9 and Propositions 10, 11.

Corollary 12. $\left\{\varphi^{-1 / 2}(r) c_{r}\right\}_{r \geq 1}$ is orthonormal and complete in $\mathcal{B}^{1}$.

Corollary 13. If $f \in \mathcal{B}^{q}(q \geq 1)$ and $M\left(f \bar{c}_{r}\right)=0$ for every $r \in \mathbb{N}$, then $\|f\|_{q}=0$.

In 1976 Schwarz and Spilker [16] proved Corollary 13 in the case of $q=2$ and in the case of $q=1$ for bounded $f$. In 1988 Hildebrand, Schwarz and Spilker [8] proved Theorem 16 in the case of $q=2$ and noticed that the 
theorem also holds for $q=1$ and $f \in \mathcal{B}^{1}$ bounded (unpublished). I have been informed by K. H. Indlekofer that Theorem 16 is already known in the general case, but it does not seem to be published yet.

Lemma 14. If $f \in \mathcal{B}^{q}(q \geq 1)$ and $s \in \mathbb{N}$, then

$$
\sum_{r \mid s} \hat{f}^{\mathrm{R}} \varphi^{-1 / 2}(r) c_{r}^{*}=S_{M_{t}} f^{*}
$$

on some $R$-Vilenkin group, $s=M_{t}$.

Let $s: \mathbb{N} \rightarrow \mathbb{N}$ be a sequence of natural numbers. Consider the condition

(1) For each $k \in \mathbb{N}$ there exists an $n=n(k)$ such that $k \mid s\left(n^{\prime}\right)$ for all $n^{\prime} \geq n$.

Theorem 15. If $f \in \mathcal{B}^{u}$ and the sequence $s: \mathbb{N} \rightarrow \mathbb{N}$ satisfies condition (1), then $K_{s} f(n)$ converges to $f(n)$, uniformly in $n$.

Theorem 16. If $f \in \mathcal{B}^{q}(q \geq 1)$ and the sequence $s: \mathbb{N} \rightarrow \mathbb{N}$ satisfies condition (1), then $K_{s} f\|\cdot\|_{q}$-converges to $f$.

We now define the modulus of continuity of arithmetical functions. The origin of the definition is in Vilenkin analysis.

Definition 17. Let $f \in D$. The $\|\cdot\|_{u}$-modulus of continuity and $\|\cdot\|_{q}$-modulus of continuity of $f(q \geq 1)$ are defined by

$$
\begin{aligned}
\omega_{n}^{q}(f) & :=\sup _{p \in \mathbb{N}}\left\|f\left(\cdot \oplus p M_{n}\right)-f(\cdot)\right\|_{q}, \\
\omega_{n}(f) & :=\sup _{p \in \mathbb{N}} \sup _{j \in \mathbb{N}}\left|f\left(j \oplus p M_{n}\right)-f(j)\right|,
\end{aligned}
$$

where $n \in \mathbb{N}_{0}$ and $G_{m}$ is some fixed $\mathrm{R}$-Vilenkin group.

We define the corresponding best approximation of $f$ by trigonometric polynomials as follows:

$$
\begin{aligned}
& E_{n}^{q}(f):=\inf _{\left\{c_{k}\right\}}\left\|f-\sum_{k=0}^{n-1} c_{k} e^{2 \pi i \check{k}}\right\|_{q} \text { for }\|f\|_{q}<\infty, \\
& E_{n}(f):=\inf _{\left\{c_{k}\right\}}\left\|f-\sum_{k=0}^{n-1} c_{k} e^{2 \pi i \check{k}}\right\|_{u} \text { for }\|f\|_{u}<\infty,
\end{aligned}
$$

where $c_{k} \in \mathbb{C}, k, n \in \mathbb{N}_{0}$.

The following theorems show that these definitions are not unnatural.

Theorem 18. If $f \in \mathcal{B}^{q}(q \geq 1)$ (resp. $\left.f \in \mathcal{B}^{u}\right)$, then

$$
\begin{gathered}
\omega_{n}^{q}(f)\left(\text { resp. } \omega_{n}(f)\right) \downarrow 0 \quad \text { for all } R \text {-Vilenkin groups } G_{m}, \\
\left|\left\{M\left(f e^{-2 \pi i a / r}\right) \mid(a, r)=1, a \in\{1, \ldots, r\}\right\}\right|=1 .
\end{gathered}
$$


Theorem 19. Let $f \in D$. If there exists an $R$-Vilenkin group $G_{m}$ such that $\omega_{n}(f) \downarrow 0$, then $f$ is a $\|\cdot\|_{u}$-periodic arithmetical function $\left(^{1}\right)$. If (3) holds, then $f \in \mathcal{B}^{u}$.

TheOREM 20. Let $f \in D$. If there exists an $R$-Vilenkin group $G_{m}$ such that

(4) $\quad\left\|f_{n}\right\|_{1} \rightarrow 0, \quad$ where $f_{n}(j):=\left\|f(j)-M\left(f\left(j \oplus \cdot M_{n}\right)\right)\right\|_{q}^{q} \quad(q \geq 1)$,

then $f$ is a $\|\cdot\|_{q}$-periodic arithmetical function. If (3) holds, then $f \in \mathcal{B}^{q}$. If $f \in \mathcal{B}^{q}$, then $\left\|f_{n}\right\|_{1} \downarrow 0$ on each $R$-Vilenkin $G_{m}$.

Theorem 21. If $f \in \mathcal{B}^{q}(q \geq 1)$ (resp. $\left.f \in \mathcal{B}^{u}\right)$, then

$$
\begin{gathered}
E_{M_{n}}^{q}(f) \leq\left\|K_{M_{n}} f-f\right\|_{q} \leq \omega_{n}^{q}(f) \leq 2 E_{M_{n}}^{q}(f), \\
\text { (resp. } \left.E_{M_{n}}(f) \leq\left\|K_{M_{n}} f-f\right\|_{u} \leq \omega_{n}(f) \leq 2 E_{M_{n}}(f)\right),
\end{gathered}
$$

where $G_{m}$ is any fixed $R$-Vilenkin group.

Corollary 22. Let $r \in \mathbb{N}, f \in \mathcal{B}^{q}(q \geq 1)$ (resp. $\left.f \in \mathcal{B}^{u}\right)$. Then for all $a, b \in \mathcal{B}_{r}$,

$$
\left\|K_{r} f-f\right\|_{q} \leq 2\|a-f\|_{q} \quad\left(\text { resp. }\left\|K_{r} f-f\right\|_{u} \leq 2\|b-f\|_{u}\right) .
$$

In the case of $q=2$, Corollary 22 with constant 1 is proved in [8] by Hildebrand, Schwarz and Spilker. Their method does not seem to work in the general case. It is also possible that (5) does not hold without the constant 2 for all $q$.

The following theorem for $C\left(G_{m}\right)$ and $L^{q}\left(G_{m}\right)(q=1,2)$ is proved by Rubinshteĭn [11] and for arbitrary $q \geq 1$ by Fridli [3].

TheOREM 23. Let $G_{m}$ be an $R$-Vilenkin group and let $z_{n} \downarrow 0, q \geq 1$. There exists an $f \in \mathcal{B}^{q}$ and also a $g \in \mathcal{B}^{u}$ for which $\omega_{n}^{q}(f)=\omega_{n}(g)=z_{n}$ for each $n \in \mathbb{N}_{0}$.

Denote by

$$
S_{n}^{\mathrm{R}}(f):=\sum_{r=1}^{n} \hat{f}^{\mathrm{R}}(r) c_{r} \varphi^{-1 / 2}(r) \quad\left(f \in \mathcal{B}^{1}\right)
$$

the $n$th partial sum of the Ramanujan series of $f$. Theorem 24 is proved for limit periodic arithmetical functions in [5], and our version is a trivial consequence.

$\left({ }^{1}\right)$ The Banach space of $\|\cdot\|_{x}$-periodic $(x=q$ or $x=u)$ arithmetical functions is the closure of $B\left(B:=\bigcup_{k \in \mathbb{N}} B_{k}, B_{k}\right.$ is the set of $\bmod k$ periodic arithmetical functions $)$ in $\left\{f \in D \mid\|f\|_{x}<\infty\right\}$ with respect to $\|\cdot\|_{x}$. 
Theorem 24. Let $f \in \mathcal{B}^{q}, 1 \leq q \leq 2, p^{-1}+q^{-1}=1$, and let $G_{m}$ be an $R$-Vilenkin group. If

$$
A:=\sum_{k=0}^{\infty} M_{k}^{1-1 / p} m_{k} \ln m_{k} \omega_{k}^{q}(f)<\infty
$$

then

$$
c_{q} A>\sum_{\alpha \in \mathbb{Q}}\left|M\left(f e^{-2 \pi i \alpha}\right)\right|,
$$

thus $S_{n}^{\mathrm{R}} f$ uniformly converges to $f(n \rightarrow \infty)$.

In [5] Theorem 4 is proved for $\|\cdot\|_{q}$-limit periodic arithmetical functions $(q>1)$, hence it also holds for $f \in \mathcal{B}^{q}(q>1)$. An easy consequence is that $f \in \mathcal{B}^{q}(q>1)$ implies

$$
S_{n} f \stackrel{\|\cdot\|_{q}}{\longrightarrow} f
$$

This fails to hold for $q=1$.

TheOrem 25. Let $G_{m}$ be an $R$-Vilenkin group. There exists an $f \in \mathcal{B}^{1}$ such that $\sup _{n \in \mathbb{N}}\left\|S_{n} f\right\|_{1}=\infty$.

Simon [17] proved that for each Vilenkin group $G_{m}$ there exists an $F \in L^{1}\left(G_{m}\right)$ such that $S_{n} F$ diverges everywhere. Does this hold for $\mathcal{B}^{1}$ functions?

Most interesting is the case of a $\mathcal{B}^{q}(q>1)$ because Hildebrand [7] proved the existence of a $\mathcal{B}^{q}$ almost even arithmetical function whose Ramanujan expansion converges to plus infinity everywhere. But Gosselin [6] and Schipp [13] proved on bounded Vilenkin groups $\left(\sup m_{s}<\infty\right.$, of course in this case $G_{m}$ is not R-Vilenkin) the $\mu$-almost everywhere convergence of $S_{n} F$ for $F \in L^{q}\left(G_{m}\right)(q>1)$. What can be said of the convergence of $S_{n} f^{*}$ in the case of $f \in \mathcal{B}^{q}(q>1)$ ?

The theorem of Gosselin and Schipp is an open question for unbounded $G_{m}$ groups $\left(\sup m_{s}=\infty, G_{m}\right.$ not necessarily R-Vilenkin of course; the origin of this topic is Luzin's conjecture, and Carleson's and Hunt's results), therefore it would be interesting to construct (if possible) a counterexample by Hildebrand's method.

Here we remark that the author proved the existence of a $\|\cdot\|_{u}$ (uniform) limit periodic arithmetical function such that

$$
S_{n} f^{*}(\check{j})=\sum_{k=0}^{n-1} M\left(f e^{-2 \pi i \check{k}}\right) e^{-2 \pi i \check{k} j}
$$

diverges for each $j \in \mathbb{N}$ (the proof will be published elsewhere). 


\section{Proofs}

Proof of Theorem 5. For every $0<\varepsilon$ there exists an $f_{\varepsilon} \in \mathcal{B}_{k(\varepsilon)}$ such that $\left\|f-f_{\varepsilon}\right\|_{u}<\varepsilon$. Since $f_{\varepsilon}$ is even, it is easy to see that there exists a unique continuous step function $f_{\varepsilon}^{*}: G_{m} \rightarrow \mathbb{C}$ such that $f_{\varepsilon}(n)=f_{\varepsilon}^{*}(\check{n})$ for all $n \in \mathbb{N}$ and $M\left(f_{\varepsilon}\right)=\int_{G_{m}} f_{\varepsilon}^{*} d \mu$.

The limit $f^{*}(x):=\lim _{\varepsilon \rightarrow 0} f_{\varepsilon}^{*}(x)$ exists for all $x \in G_{m}$.

Indeed, let $\varepsilon_{1}, \varepsilon_{2}>0$. Set

$$
k^{*}:=\min _{n \in \mathbb{N}}\left(n \in \mathbb{N}: k \mid M_{n}\right) .
$$

Take an $x \in G_{m}$. If $x=x_{\varepsilon_{1}}+x^{\prime}=x_{\varepsilon_{2}}+x^{\prime \prime}$, where $x^{\prime} \in I_{M_{k^{*}\left(\varepsilon_{1}\right)}}$ and $x^{\prime \prime} \in I_{M_{k^{*}\left(\varepsilon_{2}\right)}}$, then for the step functions $f_{\varepsilon_{1}}^{*}, f_{\varepsilon_{2}}^{*}$ we have

$$
\left|f_{\varepsilon_{1}}^{*}(x)-f_{\varepsilon_{2}}^{*}(x)\right|=\left|f_{\varepsilon_{1}}\left(\check{x}_{\varepsilon_{1}}\right)-f_{\varepsilon_{2}}\left(\check{x}_{\varepsilon_{2}}\right)\right| .
$$

Since $x-x_{\varepsilon_{i}} \in I_{M_{k^{*}\left(\varepsilon_{i}\right)}}(i=1,2)$, supposing $k^{*}\left(\varepsilon_{1}\right) \leq k^{*}\left(\varepsilon_{2}\right)$ we find that

$$
M_{k^{*}\left(\varepsilon_{1}\right)} \mid \check{x}_{\varepsilon_{1}}-\check{x}_{\varepsilon_{2}},
$$

hence

$$
f_{\varepsilon_{1}}\left(\check{x}_{\varepsilon_{1}}\right)=f_{\varepsilon_{1}}\left(\check{x}_{\varepsilon_{2}}\right) \text {. }
$$

This implies that

$$
\begin{aligned}
\left|f_{\varepsilon_{1}}^{*}\left(\check{x}_{\varepsilon_{1}}\right)-f_{\varepsilon_{2}}^{*}\left(\check{x}_{\varepsilon_{2}}\right)\right| & =\left|f_{\varepsilon_{1}}\left(\check{x}_{\varepsilon_{2}}\right)-f_{\varepsilon_{2}}\left(\check{x}_{\varepsilon_{2}}\right)\right| \\
& \leq\left\|f-f_{\varepsilon_{1}}\right\|_{u}+\left\|f-f_{\varepsilon_{2}}\right\|_{u}<\varepsilon_{1}+\varepsilon_{2} .
\end{aligned}
$$

This shows the uniform convergence of the continuous step functions $f_{\varepsilon}^{*}$ to $f^{*}$ on the R-Vilenkin group $G_{m}$. Since $G_{m}$ is compact, $f^{*}$ is also continuous. Since $\{\check{n} \mid n \in \mathbb{N}\}$ is dense in $G_{m}$, the unicity of $f^{*}$ is proved. We have

$$
\int_{G_{m}} f^{*} d \mu=\lim _{\varepsilon \rightarrow 0} \int f_{\varepsilon}^{*} d \mu .
$$

That is, $M\left(f_{\varepsilon}\right)$ converges as $\varepsilon \rightarrow 0$. We have

$$
\begin{aligned}
M\left(f_{\varepsilon}^{0}\right)-\varepsilon & \leq \underline{M}\left(f_{\varepsilon}^{0}\right)-\bar{M}\left(\left|f^{0}-f_{\varepsilon}^{0}\right|\right) \leq \underline{M}\left(f^{0}\right) \leq \bar{M}\left(f^{0}\right) \\
& \leq \bar{M}\left(\left|f^{0}-f_{\varepsilon}^{0}\right|\right)+\bar{M}\left(f_{\varepsilon}^{0}\right) \leq \varepsilon+M\left(f_{\varepsilon}^{0}\right)
\end{aligned}
$$

$\left(g^{0}=\operatorname{Re} g\right.$ or $\left.\operatorname{Im} g, g=f, f_{\varepsilon}\right)$.

Thus $\int_{G_{m}} f_{\varepsilon}^{*} d \mu=M\left(f_{\varepsilon}\right)$ implies that $\int f^{*} d \mu=M(f)$. If $n \in \mathbb{N}$, then

$$
f^{*}(\check{n})=\lim _{\varepsilon \rightarrow 0} f_{\varepsilon}^{*}(\check{n})=\lim _{\varepsilon \rightarrow 0} f_{\varepsilon}(n) .
$$

The proof of Theorem 5 is complete.

Proof of Theorem 6. For each $\varepsilon>0$, there exists an $f_{\varepsilon} \in \mathcal{B}_{k(\varepsilon)}$ such that $\left\|f-f_{\varepsilon}\right\|_{q}<\varepsilon$. Thus $f_{\varepsilon_{i}}^{*}\left(0<\varepsilon_{i} \rightarrow 0\right)$ is a Cauchy sequence in 
$L^{q}\left(G_{m}\right)$. Hence there exists a unique $f^{*} \in L^{q}\left(G_{m}\right)$ such that

$$
\lim _{\varepsilon_{i} \rightarrow 0}\left\|f^{*}-f_{\varepsilon_{i}}^{*}\right\|_{L^{q}}=\lim _{\varepsilon_{i} \rightarrow 0}\left\|f-f_{\varepsilon_{i}}\right\|_{q}=0
$$

(uniqueness in the sense of equality $\mu$-a.e.).

The proof of Theorem 6 is complete.

Theorem 7 can be proved by the method of W. Schwarz (Proposition 3.2 in [16]). The proof of Theorem 8 can be found in [5]. Theorem 9 is proved for $\|\cdot\|_{q}$-limit periodic arithmetical functions $(q \geq 1)$ in $[5]$ hence it also holds for $\mathcal{B}^{q}(q \geq 1)$ functions.

Proof of Proposition 10. If $a / r=1$ and $(a, r)=1$, then $a=$ $r=1$. Since $c_{1}^{*}=\kappa_{0}=1$, we can suppose $a / r<1<r$. Let $n$ be the least natural number for which $a M_{n} / r \in \mathbb{N}$. If $k$ is an integer in $\left[0, M_{n}\right)$, then $k=k_{n-1} M_{n-1}+\ldots+k_{0} M_{0}$. This gives $\check{k}=M_{n}^{-1}\left(k_{0} m_{n-1} \ldots m_{1}+\right.$ $\left.k_{1} m_{n-1} \ldots m_{2}+\ldots+k_{n-1}\right)=: M_{n}^{-1} k^{\prime}$. It is easy to see that $k^{\prime}$ can be any integer in $\left[0, M_{n}\right)$, hence there exists a unique $k \in\left[0, M_{n}\right)$ such that $k^{\prime}=a M_{n} / r$, thus $\check{k}=a / r$. From Theorem 8 it follows that

$$
c_{r}^{*}=\sum_{\substack{a=1 \\(a, r)=1}}^{r} \kappa_{(a / r)^{\vee}} .
$$

The proof of Proposition 10 is complete.

Proof of Proposition 11. Let $\varepsilon>0$. There exists an $f_{\varepsilon} \in \mathcal{B}$ such that

$$
\varepsilon>\left\|f-f_{\varepsilon}\right\|_{q}=\left\|f^{*}-f_{\varepsilon}^{*}\right\|_{L^{q}} \quad(\text { Theorem } 6) .
$$

Since $f_{\varepsilon} \in \mathcal{B}_{j}$ for some $j \in \mathbb{N}$, by Cohen's theorem

$$
f_{\varepsilon}=\sum_{r \mid j} \beta_{r} c_{r} \varphi^{-1 / 2}(r)
$$

Hence

$$
f_{\varepsilon}^{*}=\sum_{r \mid j} \beta_{r} c_{r}^{*} \varphi^{-1 / 2}(r), \quad \text { where } \beta_{r}=\hat{f}_{\varepsilon}^{\mathrm{R}}(r) .
$$

This obviously gives the proof for the function $f_{\varepsilon}$. Now,

$$
\begin{aligned}
\left|\hat{f}^{*}(n)-\varphi^{-1 / 2}(r) \hat{f}^{\mathrm{R}}(r)\right| \leq & \left|\hat{f}^{*}(n)-\hat{f}_{\varepsilon}^{*}(n)\right|+\left|\hat{f}_{\varepsilon}^{*}(n)-\hat{f}_{\varepsilon}^{\mathrm{R}}(r) \varphi^{-1 / 2}(r)\right| \\
& +\varphi^{-1 / 2}(r)\left|\hat{f}_{\varepsilon}^{\mathrm{R}}(r)-\hat{f}^{\mathrm{R}}(r)\right| \\
\leq & 2 \int_{G_{m}}\left|f^{*}-f_{\varepsilon}^{*}\right| d \mu \leq 2\left\|f^{*}-f_{\varepsilon}^{*}\right\|_{L^{q}}<2 \varepsilon .
\end{aligned}
$$

The proof of Proposition 11 is complete.

Proof of Lemma 14. First we give the construction of the desired R-Vilenkin group. Let $m_{0}, m_{1}, \ldots, m_{t-1}$ be integers not less than 2 with 
$m_{0} m_{1} \ldots m_{t-1}=s$. The $m_{i}$ 's for $i \geq t$ are defined in such a way that $G_{m}$ is an $\mathrm{R}$-Vilenkin group. Then obviously

$$
\{a / r|(a, r)=1, a \in\{1, \ldots, r\}, r| s\}=\{b / s \mid b \in\{1, \ldots, s\}\} .
$$

Let $b / s$ belong to the right side of $(6), b \neq s$. We have $b / s=b / M_{t}$, hence $\left((b / s)^{\vee}\right)^{\prime}=b$. Since the set of $b$ 's is $\{1, \ldots, s-1\}=\left\{1, \ldots, M_{t}-1\right\}$, the set of $\left(b / M_{t}\right)^{\vee}=(b / s)^{\vee}$ is also $\left\{1, \ldots, M_{t}-1\right\}$. We have $c_{1}^{*}=\kappa_{0}=1$. By the application of Propositions 10, 11 the proof of Lemma 14 is complete.

Proof of Theorem 15. Use the result of Lemma 14 and apply Theorem 2. Let $\varepsilon>0$ and $f_{\varepsilon} \in \mathcal{B}_{k(\varepsilon)}$ such that $\left\|f-f_{\varepsilon}\right\|_{u}<\varepsilon$. By (1) there exists an $n_{\varepsilon}$ such that $k(\varepsilon) \mid s(n)$ for $n \geq n_{\varepsilon}$. Lemma 14 and Theorems 2 and 5 give

$$
\begin{aligned}
\left|K_{s(n)} f(j)-f(j)\right| & =\left|S_{M_{t(n)}} f^{*}(\check{j})-f^{*}(\check{j})\right| \\
& =M_{t(n)} \int_{I_{t(n)}(\check{j})}\left|f^{*}(x)-f^{*}(\check{j})\right| d \mu(x) .
\end{aligned}
$$

Since $f^{*}$ is uniformly continuous on the compact set $G_{m}$,

$$
\begin{aligned}
\sup _{x \in I_{t}(\breve{j})} & \left|f^{*}(x)-f^{*}(\check{j})\right| \\
& =\sup _{x \in I_{t}(\breve{j}) \cap \mathbb{Q}_{m}}\left|f^{*}(x)-f^{*}(\check{j})\right|=\sup _{k \equiv j\left(\bmod M_{t(n)}\right)}|f(k)-f(j)| \\
& \leq 2\left\|f-f_{\varepsilon}\right\|_{u}+\sup _{k \equiv j\left(\bmod M_{t}\right)}\left|f_{\varepsilon}(k)-f_{\varepsilon}(j)\right|=2\left\|f-f_{\varepsilon}\right\|_{u}<2 \varepsilon .
\end{aligned}
$$

Thus $\left|K_{s(n)} f(j)-f(j)\right|<2 \varepsilon$.

This completes the proof of Theorem 15 .

Proof of Theorem 16. Since $f \in \mathcal{B}^{q}(q \geq 1), f^{*} \in L^{q}\left(G_{m}\right)$. Fix an $\varepsilon>0$. There exists an $f_{\varepsilon} \in \mathcal{B}_{k(\varepsilon)}$ for which $\left\|f-f_{\varepsilon}\right\|_{q}<\varepsilon$. (1) implies the existence of an $n_{\varepsilon} \in \mathbb{N}$ such that $k(\varepsilon) \mid s(n)$ for all $n_{\varepsilon} \leq n \in \mathbb{N}$. Fix such an $s=s(n)$ and consider the Vilenkin group given by Lemma 14. Cohen's theorem gives

$$
f_{\varepsilon}^{*}=\sum_{r \mid k} \beta_{r} c_{r}^{*} \quad\left(\beta_{r}=\varphi^{-1}(r) M\left(f \bar{c}_{r}\right)\right) .
$$

Since by Lemma $14,\left(K_{s} g\right)^{*}=S_{M_{t}} g^{*}$ for all $g \in \mathcal{B}^{1}$, Theorem 1 , Propositions 10, 11 and Lemma 14 give $\left(K_{s} f_{\varepsilon}\right)^{*}=S_{M_{t}} f_{\varepsilon}^{*}=f_{\varepsilon}^{*}$. This and Theorems 6 and 3 imply

$$
\begin{aligned}
\left\|K_{s} f-f\right\|_{q} & =\left\|S_{M_{t}} f^{*}-f^{*}\right\|_{L^{q}} \leq\left\|S_{M_{t}} f^{*}-f_{\varepsilon}^{*}\right\|_{L^{q}}+\left\|f-f_{\varepsilon}\right\|_{q} \\
& =\left\|S_{M_{t}}\left(f^{*}-f_{\varepsilon}^{*}\right)\right\|_{L^{q}}+\left\|f-f_{\varepsilon}\right\|_{q} \\
& <\left(A_{q}+1\right)\left\|f-f_{\varepsilon}\right\|_{q}<\left(A_{q}+1\right) \varepsilon .
\end{aligned}
$$

The proof of Theorem 16 is complete. 
Proof of Theorem 18 . Theorems 6 and 5 give respectively

$$
\begin{gathered}
\omega_{n}^{q}(f)=\sup _{y \in I_{n}}\left\{\int_{G_{m}}\left|f^{*}(x+y)-f^{*}(x)\right|^{q} d \mu(x)\right\}^{1 / q}, \\
\omega_{n}(f)=\sup _{y \in I_{n}} \sup _{x \in G_{m}}\left|f^{*}(x+y)-f^{*}(x)\right| .
\end{gathered}
$$

The right side of (7) is the usual $L^{q}\left(G_{m}\right)$-modulus of continuity of $f^{*}$. The right side of (8) is the $C\left(G_{m}\right)$-modulus of continuity of $f^{*}$. Thus $\omega_{n}^{q}(f)$ (resp. $\omega_{n}(f) \downarrow 0$, directly from Vilenkin analysis [1]. (3) is easy to verify. The proof is complete.

Proof of Theorem 19. Let $\varepsilon>0$ be fixed. For $n>n_{0}(\varepsilon)$,

$$
\omega_{n}(f)=\sup _{p \in \mathbb{N}} \sup _{j \in \mathbb{N}}\left|f(j)-f\left(j \oplus p M_{n}\right)\right|<\varepsilon,
$$

that is,

$$
\left|f(j)-f\left(j \oplus p M_{n}\right)\right|<\varepsilon \quad \text { for all } j, p \in \mathbb{N} .
$$

Define (with $f(0):=0$ )

$$
\hat{f}_{c}(k):=M_{c}^{-1} \sum_{a=0}^{M_{c}-1} f(a) \bar{\kappa}_{k}(\check{a}) \quad \text { and } \quad g(j):=\sum_{k=0}^{M_{n}-1} \hat{f}_{c}(k) \bar{\kappa}_{k}(\check{j}) \quad\left(c \in \mathbb{N}_{0}\right) \text {. }
$$

Then $g$ is periodic. Suppose that $c>n$. Then

$$
\begin{aligned}
|f(j)-g(j)| & =\left|f(j)-M_{c}^{-1} \sum_{a=0}^{M_{c}-1} f(a) \sum_{k=0}^{M_{n}-1} \bar{\kappa}_{k}(\check{a}) \kappa_{k}(\check{j})\right| \\
& =\left|f(j)-\frac{M_{n}}{M_{c}} \sum_{\substack{a=0 \\
a \equiv j\left(\bmod M_{n}\right)}}^{M_{c}-1} f(a)\right| \quad \text { (Theorem 2) } \\
& \leq \frac{M_{n}}{M_{c}} \sum_{p=0}^{M_{c} / M_{n}-1}\left|f(j)-f\left(\sum_{i=0}^{n-1} j_{i} M_{i} \oplus p M_{n}\right)\right|<2 \varepsilon .
\end{aligned}
$$

That is, $f$ is uniform limit periodic.

In [5] it is proved for uniform limit periodic arithmetical functions that $S_{M_{n}} f^{*} \stackrel{\|\cdot\|_{u}}{\longrightarrow} f$. The rest of the proof of Theorem 19 follows from $S_{M_{n}} f^{*}=$ $\sum_{r \mid M_{n}} M\left(f \bar{c}_{r}\right) c_{r} \varphi^{-1}(r)$ as (3) is satisfied for $f$. The proof is complete.

Proof of Theorem 20. Suppose that $f \in \mathcal{B}^{q}$. Then

$$
\begin{aligned}
& \left\{\int_{G_{m}} M_{n} \int_{I_{n}}\left|f^{*}(x+h)-f^{*}(x)\right|^{q} d \mu(h) d \mu(x)\right\}^{1 / q} \\
& =M_{n}^{1 / q}\left\{\int_{G_{m}} \int_{I_{n}}\left|f^{*}(x+h)-f^{*}(x)\right|^{q} d \mu(h) d \mu(x)\right\}^{1 / q}
\end{aligned}
$$




$$
\begin{aligned}
& =M_{n}^{1 / q}\left\{\int_{I_{n}} \int_{G_{m}}\left|f^{*}(x+h)-f^{*}(x)\right|^{q} d \mu(x) d \mu(h)\right\}^{1 / q} \quad \text { (Fubini's theorem) } \\
& =\left\{M_{n} \int_{I_{n}} \int_{G_{m}}\left|f^{*}(x+h)-f^{*}(x)\right|^{q}\right\}^{1 / q} \\
& \leq\left\{\sup _{h \in I_{n}}\left\|f^{*}(x+h)-f^{*}(x)\right\|_{q}^{q}\right\}^{1 / q}=\omega_{n}^{q}(f) \downarrow 0
\end{aligned}
$$

by Theorem 18. Since

$$
\left\|f_{n}\right\|_{1}^{q^{-1}}=\left\{\int_{G_{m}} M_{n} \int_{I_{n}}\left|f^{*}(x+h)-f^{*}(x)\right|^{q} d \mu(h) d \mu(x)\right\}^{1 / q},
$$

the last statement of Theorem 20 is proved. The other two statements being trivial, the proof is complete.

Corollary 22 is a straightforward consequence of Theorem 21, which can be proved by the application of the similar result for $\psi \alpha$ systems on Vilenkin groups [4].

The proof of Theorem 24 can also be obtained with the help of the similar result for $\psi \alpha$ systems [4], which generalizes the result of Zhantlesov [19] proved in the case of the original Vilenkin system (i.e. $\alpha=1$ ).

Proof of Theorem 23. The original idea, concerning Vilenkin (and not necessarily R-Vilenkin) groups comes from Fridli [3] and Rubinshtein [11]. They prove the existence of $L^{q}\left(G_{m}\right)$ and $C\left(G_{m}\right)$ functions whose $\omega_{n}^{q}$ respectively $\omega_{n}$ modulus of continuity is $z_{n}$. In [5] (Theorem 25) it is proved that there exists a $\|\cdot\|_{1}$-limit periodic arithmetical function $f: \mathbb{N} \rightarrow \mathbb{N}$ such that $\omega_{n}^{q}(f)=z_{n}$ for each $n \in \mathbb{N}_{0}$ and $f^{*}=F \mu$-almost everywhere on $G_{m}$, where $F \in L^{q}\left(G_{m}\right)$ satisfies the following relation: $\left\|F_{n}-F\right\|_{L^{q}} \rightarrow 0$ $(n \rightarrow+\infty)$, where

$$
F_{n}=\sum_{i=0}^{\infty} \alpha_{n, i} \operatorname{char}_{I_{i} \backslash I_{i+1}}
$$

( $n \in \mathbb{N}_{0}$, $\operatorname{char}_{B}$ denotes the characteristic function of the set $B, \alpha_{n, i}$ is some complex number, $n, i \in \mathbb{N}_{0}$ ).

Define arithmetical functions

$$
f_{n}:=\sum_{i=0}^{\infty} \alpha_{n, i} \beta_{i}
$$

where

$$
\beta_{i}(j):= \begin{cases}1 & \text { if } M_{i} \mid j \text { and } M_{i+1} \nmid j \\ 0 & \text { otherwise } \quad\left(\beta_{i}(0):=0\right) .\end{cases}
$$


It suffices to show that

$$
\beta_{i} \text { is an even arithmetical function, and } f_{n} \in \mathcal{B}^{q},
$$

$$
f_{n}^{*}=F_{n} \quad \mu \text {-a.e. on } G_{m} .
$$

Indeed, by (9) and (10) Theorem 6 gives that the arithmetical function $f$, whose $\|\cdot\|$-modulus of continuity is $z$, is a $\|\cdot\|_{q}$-almost even arithmetical function, that is, the proof of Theorem 23 would be complete. Now,

$$
\begin{aligned}
& M\left(\beta_{k}(\cdot) e^{-2 \pi i(a / n)} \cdot\right) \\
& =\lim _{b \rightarrow+\infty} b^{-1} \sum_{j=0}^{b-1} \beta_{k}(j) e^{-2 \pi i(a / n) j}=\left(M_{k+1} n\right)^{-1} \sum_{j=0}^{M_{k+1} n} \beta_{k}(j) e^{-2 \pi i(a / n) j} \\
& =\left(M_{k+1} n\right)^{-1} \sum_{j=0}^{M_{k+1} n} e^{-2 \pi i(a / n) j}-\left(M_{k+1} n\right)^{-1} \sum_{j=0}^{M_{k+1} n} e^{-2 \pi i(a / n) j} \\
& =: A_{1}-A_{2} \quad(a, n \in \mathbb{N},(a, n)=1) .
\end{aligned}
$$

It is easy to see that

$$
A_{1}=\left\{\begin{array}{ll}
M_{k}^{-1} & \text { if } n \mid M_{k}, \\
0 & \text { otherwise }
\end{array} \quad A_{2}= \begin{cases}M_{k+1}^{-1} & \text { if } n \mid M_{k+1} \\
0 & \text { otherwise }\end{cases}\right.
$$

therefore $A_{1}-A_{2}$ does not depend on $a$.

Hence, as $\beta_{k}$ is periodic modulo $M_{k+1}, \beta_{k}$ is even. Define

$f_{n}^{K}:=\sum_{i=0}^{K} \alpha_{n, i} \beta_{i}, \quad F_{n}^{K}:=\sum_{i=0}^{K} \alpha_{n, i} \operatorname{char}_{I_{i} \backslash I_{i+1}} \quad\left(f_{n}^{K} \in D, F_{n}^{K}: G_{m} \rightarrow \mathbb{C}\right)$.

We have

$$
\left\|f_{n}-f_{n}^{K}\right\|_{q}=\left\{\sum_{i=K+1}^{\infty}\left|\alpha_{n, i}\right|^{q}\left(1 / M_{i}-1 / M_{i+1}\right)\right\}^{1 / q}=\left\|F_{n}-F_{n}^{K}\right\|_{L^{q}} \rightarrow 0
$$

as $K \rightarrow \infty$ because $F_{n} \in L^{q}\left(G_{m}\right)$.

Since $f_{n}^{K}$ is even, $f_{n}$ is almost even. We have $\left\|f_{n}\right\|_{q}=\left\|F_{n}\right\|_{L^{q}}$, consequently $f_{n} \in \mathcal{B}^{q}$. Next, $\beta_{i}^{*}=\operatorname{char}_{I_{i} \backslash I_{i+1}} \mu$-almost everywhere on $G_{m}$, thus $\left(f_{n}^{K}\right)^{*}=F_{n}^{K} \mu$-a.e. on $G_{m}$ for each $k \in \mathbb{N}_{0}$. This implies that $f_{n}^{*}=F_{n} \mu$-a.e. on $G_{m}$, that is, (9) and (10) are proved. Thus the proof of Theorem 23 for the $\|\cdot\|_{q}$-modulus of continuity is complete. To prove the existence of a uniform almost even arithmetical function whose $\|\cdot\|_{u}$-modulus of continuity is a given sequence, one can apply the idea of Rubinshteln [11] and the methods used above. 
Proof of Theorem 25. Define arithmetical functions

$$
a_{n}(j):= \begin{cases}M_{n+1}-M_{n} & \text { if } M_{n+1} \mid j, \\ -M_{n} & \text { if } M_{n} \mid j \text { and } M_{n+1} \nmid j \quad\left(a_{n}(0):=0, n \in \mathbb{N}_{0}\right), \\ 0 & \text { if } M_{n} \nmid j .\end{cases}
$$

Then

$$
a_{n}(j)=D_{M_{n+1}}(j, 0)-D_{M_{n}}(j, 0)=\sum_{k=M_{n}}^{M_{n+1}-1} \exp (2 \pi i \check{k} j)
$$

is even. Let $M_{n} \leq b<2 M_{n}$. Thus $b=M_{n}+b_{n-1} M_{n-1}+\ldots+b_{0} M_{0}$ and

$$
D_{b}(r, j)=D_{M_{n}}(r, j)+\sum_{k=M_{n}}^{b-1} \exp (2 \pi i \check{k}(r-j)) .
$$

Since $k=M_{n}+k_{n-1} M_{n-1}+\ldots+k_{0} M_{0}=: M_{n}+k^{-}$therefore $\check{k}=1 / M_{n+1}+$ $\left(k^{-}\right)^{\vee}$. This implies that

$D_{b}(r, j)=D_{M_{n}}(r, j)+\exp \left(2 \pi i \frac{1}{M_{n}}(r-j)\right) \sum_{k^{-}=0}^{b-M_{n}-1} \exp \left(2 \pi i\left(k^{-}\right)^{\vee}(r-j)\right)$.

Now

$$
\begin{aligned}
S_{b} a_{n}(r)= & M\left\{\sum _ { k = M _ { n } } ^ { M _ { n + 1 } - 1 } \operatorname { e x p } ( 2 \pi i \check { k } j ) \left\{\sum_{k=0}^{M_{n}-1} \exp (2 \pi i \check{k}(r-j))\right.\right. \\
& \left.\left.+\sum_{k=M_{n}}^{b-1} \exp (2 \pi i \check{k}(r-j))\right\}\right\} \\
= & \sum_{k=M_{n}}^{b-1} \exp (2 \pi i \check{k} r)=\exp \left(2 \pi i \frac{1}{M_{n+1}} r\right) D_{b-M_{n}}(r, 0) .
\end{aligned}
$$

Hence

$$
M_{n}^{-1} \sum_{b=M_{n}+1}^{2 M_{n}}\left\|S_{b} a_{n}\right\|_{1}=M_{n}^{-1} \sum_{k=1}^{M_{n}}\left\|D_{k}(\cdot, 0)\right\|_{1}=: D_{n}^{0} .
$$

Next we give a lower bound for $D_{n}^{0}$ :

$$
D_{n}^{0} \geq M_{n}^{-1} \sum_{s=0}^{n-1} M\left\{\sum_{k=1}^{M_{n}}\left|D_{k}(\cdot, 0)\right| \beta_{s}(\cdot)\right\} .
$$

(The arithmetical functions $\beta_{s}$ are defined in the proof of Theorem 23.) The product $\left|D_{k}(j, 0)\right| \beta_{s}(j)$ can be different from zero only in the case when $M_{s} \mid j$ and $M_{s+1} \nmid j$. In this case it is equal to $\left|D_{k}(j, 0)\right|$. Suppose that 
$k=\sum_{i=0}^{t} k_{i} M_{i}(t \leq n)$. We have

$$
\begin{aligned}
D_{k}(j, 0)= & D_{M_{t}}(j, 0)+\exp \left(2 \pi i \frac{1}{M_{t+1}} j\right) D_{M_{t}}(j, 0)+\ldots \\
& +\exp \left(2 \pi i j \frac{k_{t}-1}{M_{t+1}}\right) D_{M_{t}}(j, 0)+\exp \left(2 \pi i j \frac{k_{t}}{M_{t+1}}\right) D_{M_{t-1}}(j, 0) \\
& +\exp \left(2 \pi i j\left(\frac{k_{t}}{M_{t+1}}+\frac{1}{M_{t}}\right)\right) D_{M_{t-1}}(j, 0)+\ldots \\
& +\exp \left(2 \pi i j\left(\frac{k_{t}}{M_{t+1}}+\frac{k_{t-1}-1}{M_{t}}\right)\right) D_{M_{t-1}}(j, 0) \\
& +\exp \left(2 \pi i j\left(\frac{k_{t}}{M_{t+1}}+\frac{k_{t-1}}{M_{t}}\right)\right) D_{M_{t-2}}(j, 0)+\ldots \\
& +\exp \left(2 \pi i j\left(\frac{k_{t}}{M_{t+1}}+\ldots+\frac{k_{s+1}}{M_{s+2}}\right)\right) D_{M_{s}}(j, 0)+\ldots \\
& +\exp \left(2 \pi i j\left(\frac{k_{t}}{M_{t+1}}+\ldots+\frac{k_{1}}{M_{2}}+\frac{k_{0}-1}{M_{1}}\right)\right) D_{M_{0}}(j, 0) .
\end{aligned}
$$

Since $M_{s} \mid j$ and $M_{s+1} \nmid j$, assuming $k_{s} \geq 1$ we get

$$
\begin{aligned}
D_{k}(j, 0) & =\sum_{l=0}^{s} M_{l} \sum_{n=0}^{k_{l}-1} \exp \left(2 \pi i j\left(\frac{k_{t}}{M_{t+1}}+\ldots+\frac{k_{l+1}}{M_{l+2}}+\frac{n}{M_{l+1}}\right)\right) \\
& =M_{s} \sum_{n=0}^{k_{s}-1} \exp \left(2 \pi i j\left(\frac{k_{t}}{M_{t+1}}+\ldots+\frac{k_{s+1}}{M_{s+2}}\right)\right) .
\end{aligned}
$$

As a consequence,

$$
\left|D_{k}(j, 0)\right|=M_{s}\left|\sum_{n=0}^{k_{s}-1} \exp \left(2 \pi i j \frac{n}{M_{s+1}}\right)\right|=M_{s}\left|\sum_{n=0}^{k_{s}-1} \exp \left(2 \pi i \frac{j_{s} n}{m_{s}}\right)\right| .
$$

This gives

$$
\begin{aligned}
M & \left\{\sum_{k=1}^{M_{n}}\left|D_{k}(j, 0)\right| \beta_{s}(\cdot)\right\} \geq \sum_{k=1}^{M_{n}} M_{s} \frac{1}{M_{s+1}} \sum_{j_{s}=1}^{m_{s}-1}\left|\frac{\exp \left(2 \pi i j_{s} k_{s} / m_{s}\right)-1}{\exp \left(2 \pi i j_{s} / m_{s}\right)-1}\right| \\
& =\frac{1}{m_{s}} \sum_{\substack{k=M_{s} \\
k_{s} \geq 1}}^{M_{n}} \sum_{j=1}^{m_{s}-1} \frac{\left|\sin \left(\pi j k_{s} / m_{s}\right)\right|}{\sin \left(\pi j / m_{s}\right)} \\
& \geq \frac{1}{m_{s}} \frac{M_{n}-M_{s}}{m_{s}} \sum_{k_{s}=1}^{m_{s}-1} \sum_{j=1}^{m_{s}-1} \frac{\left|\sin \left(\pi j k_{s} / m_{s}\right)\right|}{\sin \left(\pi j / m_{s}\right)}
\end{aligned}
$$




$$
\begin{aligned}
& \geq c M_{n} m_{s}^{-2} \sum_{j=1}^{\left[m_{s} / 2\right]} \frac{m_{s}}{j} \sum_{j=1}^{m_{s}-1}\left|\sin \left(\pi j k_{s} / m_{s}\right)\right| \\
& \geq c M_{n} m_{s}^{-2} \sum_{j=1}^{\left[m_{s} / 2\right]} \frac{m_{s}}{j} j \sum_{k_{s}=1}^{\left[m_{s} /(2 j)\right]} \frac{j k_{s}}{m_{s}} \geq c M_{n} \sum_{j=1}^{\left[m_{s} / 2\right]} \frac{1}{j} \geq c M_{n} \log m_{s}
\end{aligned}
$$

for some absolute constant $c>0$ (which may vary from line to line). Substituting this last inequality into (11) we get

$$
D_{n}^{0} \geq c \sum_{s=0}^{n-1} \log m_{s}=c \log M_{n} \geq c n
$$

for some absolute constant $c>0$. This implies that there exists a $b=b\left(a_{n}\right)$ with $M_{n}<b \leq 2 M_{n}$ such that $\left\|S_{b} a_{n}\right\|_{1} \geq c n$.

Define

$$
f:=\sum_{n=0}^{\infty} \lambda_{n} a_{\nu_{n}}, \quad \text { where } \quad \lambda_{n} \in \mathbb{C}, \sum_{n=0}^{\infty}\left|\lambda_{n}\right|<\infty .
$$

Then $f^{K}:=\sum_{n=0}^{K} \lambda_{n} a_{\nu_{n}}$ is an even arithmetical function. We have

$$
\begin{aligned}
\left\|f-f^{K}\right\|_{1} & \leq c \sum_{n=K+1}^{\infty}\left|\lambda_{n}\right|\left(M_{\nu_{n}+1}-M_{\nu_{n}}\right) \frac{1}{M_{\nu_{n}+1}} \\
& \leq c \sum_{n=K+1}^{\infty}\left|\lambda_{n}\right| \rightarrow 0 \quad(K \rightarrow+\infty) .
\end{aligned}
$$

Thus $f \in \mathcal{B}^{1}$ is almost even.

If $j<n$, then $S_{b\left(a_{j}\right)} a_{n}=0$, hence in this case $\left\|S_{b\left(a_{j}\right)} a_{n}\right\|_{1} \leq c$. Finally, we get

$$
\begin{aligned}
\left\|S_{b\left(a_{\nu_{n}}\right)} f\right\|_{1} & =\left\|S_{b\left(a_{\nu_{n}}\right)}\left(\sum_{j=0}^{n} \lambda_{j} a_{\nu_{j}}\right)\right\|_{1} \geq\left\|S_{b\left(a_{\nu_{n}}\right)} a_{\nu_{n}}\right\|_{1}\left|\lambda_{n}\right|-c \sum_{k=0}^{\infty}\left|\lambda_{k}\right| \\
& \geq c \nu_{n}\left|\lambda_{n}\right|-c \sum_{k=0}^{\infty}\left|\lambda_{k}\right| .
\end{aligned}
$$

Now, take $\nu_{n}>n /\left|\lambda_{n}\right|\left(n \in \mathbb{N}_{0}\right)$ and $\lambda_{n} \neq 0\left(n \in \mathbb{N}_{0}\right)$. We get $\sup \left\|S_{n} f\right\|_{1}=$ $\infty$, that is, the proof of Theorem 25 is complete.

\section{References}

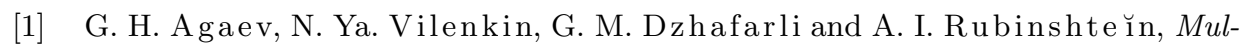
tiplicative Systems of Functions and Harmonic Analysis on 0-dimensional Groups, Izd. "ELM", Baku 1981 (in Russian). 
[2] E. Cohen, A class of arithmetical functions, Proc. Nat. Acad. Sci. U.S.A. 41 (1955), 939-944.

[3] S. Fridli, On the modulus of continuity with respect to functions defined on Vilenkin groups, Acta Math. Acad. Sci. Hungar. 45 (3-4) (1985), 393-396.

[4] G. Gát, Orthonormal systems on Vilenkin groups, ibid., to appear.

[5] -, On approximation of limit periodic arithmetical functions, Period. Math. Hungar., submitted.

[6] J. Gosselin, Almost everywhere convergence on Vilenkin-Fourier series, Trans. Amer. Math. Soc. 186 (1973), 345-370.

[7] A. Hildebrand, Über die punktweise Konvergenz von Ramanujan-Entwicklungen zahlentheoretischer Funktionen, Acta Arith. 44 (1984), 109-145.

[8] A. Hildebrand, W. Schwarz and J. Spilker, Still another proof of Parseval's equation for almost even arithmetical functions, Aequationes Math. 35 (1988), $132-139$.

[9] J. Knopfmacher, Fourier analysis of arithmetical functions, Ann. Mat. Pura Appl. 109 (1976), 177-201.

[10] —, Abstract Analytic Number Theory, North Holland, Amsterdam 1975.

[11] A. I. Rubinshte $\breve{n}$, On the modulus of continuity of functions defined on 0-dimensional groups, Mat. Zametki 23 (1978), 379-388 (in Russian).

[12] F. Schipp, On $L^{p}$-norm convergence of series with respect to product systems, Anal. Math. 2 (1978), 49-64.

[13] - , Pointwise convergence of expansions with respect to certain product systems, ibid., $65-76$.

[14] F. Schipp, W. R. Wade, P. Simon and J. Pál, Walsh Series. An Introduction to Dyadic Harmonic Analysis, Akadémiai Kiadó, Budapest, and Adam Hilger, Bristol and New York 1990.

[15] W. Schwarz und J. Spilker, Eine Anwendung des Approximationssatzes von Weierstrass-Stone auf Ramanujan-Summen, Nieuw Arch. Wisk. (3) 19 (1971), 198-209.

[16] - - -, Mean values and Ramanujan expansions of almost even arithmetical functions, in: Topics in Number Theory, Colloq. Math. Soc. J. Bolyai 13, North-Holland, Amsterdam 1976, 315-357.

[17] P. Simon, On the divergence of Vilenkin-Fourier series, Acta Math. Hungar. 41 (3-4) (1983), 359-370.

[18] N. Ya. Vilenkin, On a class of complete orthonormal systems, Izv. Akad. Nauk SSSR Ser. Mat. 11 (1947), 363-400; English transl: Amer. Math. Soc. Transl. 28 (1963), 1-35.

[19] Zh. H. Zhantlesov, On absolute convergence of Fourier series with respect to multiplicative systems, Izv. Akad. Nauk Kazakh. SSR Ser. Fiz.-Mat. 3 (1986), 14-17 (in Russian).

DEPARTMENT OF MATHEMATICS

BESSENYEI COLLEGE

P.O. BOX 166

H-4401 NYIREGYHÁZA, HUNGARY 